\title{
Development of transferable rule-sets for urban areas using QuickBird satellite imagery
}

\begin{abstract}
Although rule-based object-based classification can often perform better than the supervised approaches, its attribute selection is very time consuming and hardly transferable between different urban areas. The purpose of this study is to identify transferable rule-sets for different areas from QuickBird satellite imagery for urban areas consisting heterogeneous man-made and natural features. Object-based classification was used in this research, and a mathematical method was proposed for rule transferability for different urban areas.
\end{abstract}

Keyword: Object-based classification; Rule-based classification; Transferability; Urban areas 